\title{
Ashwagandha (Withania somnifera (L.) Dunal): A scientific review with respect to Ayurvedic perspectives
}

\section{C.P. Khare and Andrew Vikram Naharwar*}

Flat No. 507, HMM Society, Plot No. 6, Dwaraka Sector 10, New Delhi-110075, India

*Amsar Goa Pvt. Ltd., S 12 Colvale Industrial Estate, Colvale, Bardez, Goa-403513, India

\section{Article Info}

Article history

Received 11 August 2020

Revised 27 September 2020

Accepted 29 September 2020

Published online 30 December 2020

Keywords

Withania somnifera (L.) Dunal

Neuroptrotective

Immune modulator

Antitumor

Antistress

Anticancer

\begin{abstract}
Ashwagandha (Withania somnifera (L.) Dunal, Fam. Solanaceae) is one of the most important herb of Ayurveda System of Medicine used for millennia as a therapeutic tonic (Rasayana) for its wide-ranging health benefits. Among the Ayurvedic Rasayana herbs, Ashwagandha holds the most prominent place. In present review, different aspects of Ashwagandha including botanical, phytochemical and pharmacological has been covered in light of ayurvedic references has been elaborated. It discusses different vernacular names, chemotypes and details of cultivated varieties available, in addition to morphological characters, quality gradings, DNA barcoding, chemical constituents of cultivated varieties and comparison of wild and cultivated varieties. It has also included pharmacological studies reported on Ashwagandha such as neurpprotective, immunomodulatory responses, antitumor, radiosensitizing effects, antistress, adaptogenic, anticancer and toxicological profiles relevant to AYUSH. Cultivated varieties also showed hope against Alzheimer's disease. The major phytoconstituents have also been reported for their biological effects as reported. This review may be useful for academia and industry for identity confirmation and for referring other biological effects.
\end{abstract}

\section{Introduction}

India is the only country where $W$. somnifera is known as Ashwagandha, while native names of all other countries are different (Table 1). The Editors of 'The Wealth of India' (Vol. X) while writing about Ashwagandhaa of ancient Indian literature remarked that it is difficult to say whether the source of the drug (classical Ashwagandhaa) is W. somnifera.

Table 1: Vernacular names of W. somnifera in different countries

\begin{tabular}{|l|l|l|}
\hline S.No. & Vernacular name & Name of countries \\
\hline 1. & Simmi Frakh & Egypt \\
\hline 2. & $\begin{array}{l}\text { Summ-al-far, Genegeneh, Haml balbul, Bulbul } \\
\text { Baabu, Shaukaran, 'Ebab, Obeeb }\end{array}$ & Arabian Peninsul \\
Ineb ed-dib, Semm el-firakh, Foqqish, Sharma, & Africa \\
\hline 4. & Kaknaj-e-Hindi & Arabic \\
\hline 5. & Foulet el-kalb & $\begin{array}{l}\text { North Africa, Algeria, Egypt, Libya, } \\
\text { Tunisia, East Africa, Morocoo }\end{array}$ \\
\hline 6. & Geneesblaar, Meidjieblaar, Vuilsiektebos & O.F.S \\
\hline 7. & Uvimba & Fingo \\
\hline 8. & Ol-asajet & Maasai \\
\hline 9. & Kuvia & Nyamwezi \\
\hline 10. & Dambarico & Pare \\
\hline 11. & Mgeda & Sukuma \\
\hline 12. & Mosalasopeng & Sotho \\
\hline 13. & VImhepe & Swati \\
\hline 14. & Umuvuma, Umuvimba & Xhosa, Zulu \\
\hline 15. & Foakapoka & Madagascar \\
\hline
\end{tabular}

Corresponding author: Dr. C.P. Khare

Founder President, Society for New Age Herbals, Flat No. 507, HMM Society, Plot No. 6, Dwaraka Sector 10, New Delhi-110075, India E-mail: khare.herbalsociety@gmail.com

Tel.: +91-8447403217
Twenty-three Withania species are widely distributed in the drier parts of tropical and subtropical zones ranging from the Canary Islands, the Mediterranean region, and northern Africa to South West Asia. Only W. somnifera and W. coagulans are medicinally significant and cultivated in several regions.

Copyright (C) 2020 Ukaaz Publications. All rights reserved.

Email: ukaaz@yahoo.com; Website: www.ukaazpublications.com 
The Central Institute of Medicinal and Aromatic Plants (CIMAP) specified 3 chemotypes of $W$. somnifera.

- Chemotype-I (Israel) contains withaferin A as a major constituent. Minor constituents are withanolides $\mathrm{N}$ and $\mathrm{O}$.

- Chemotype-II (Israel) contains withanolide D as a major constituent. Withanolide G, 27-hydroxywithanolide D, 14alphahydroxy-withanolide D and 17alpha-hydroxywithanolide D are present in trace amounts.

- Chemotype-III (Israel) contains withanolides E-M. Leaves of offspring F (from the crossing of chemotype-I and III) contain withanolides $\mathrm{Q}$ and $\mathrm{R}$. Withanolides $\mathrm{P}$ and $\mathrm{S}$ are also present in leaves (Husain et al., 1992).

In (Lawrence) Review of Natural Products, three chemotypes have been defined.

- Chemotype-I (India) contains withanone and withaferin A as major constituents.

- Chemotype-II (Israel) contains withanolide D and 27hydroxywithanolide D as major withanolides.

- Chemotype-III (Israel) contains principally withanolide E (Dermarderosian, 2004).

Besides W. somnifera, W. coagualans, other species W. adpressa, $W$. aristata, W. frutescens, and W. obtusifolia have also been investigated for the study of their phytochemical and biological aspects. But still, these species are not well explored. For example, there are only two reports, one on each of the antifungal and cytotoxic. Three cytotoxic withanolides, $14 \alpha, 15 \alpha, 17 \beta, 20 \beta$ tetrahydroxy-1-oxo-( )-witha-2,5,24 trienolide, 22R withanolide $\mathrm{F}$ and withanolide $\mathrm{J}$ are isolated from the dichloromethane $(\mathrm{CH} \mathrm{Cl})$ fraction of the methanolic 22 extract of the leaf. But, no work has been carried on $W$. adpressa out of the phytochemical investigation of the other parts of this plant as well as on other fractions of the methanolic extract of the leaves. Hence, the other parts, viz., stem, root, fruits and flower and other fractions, viz., n-hexane, ethylacetate, n-butanol of the methanolic extract of the leaf, require chemical investigation of the compounds responsible for the cytotoxicity and other biological activities (Abdeljebbar et al., 2009).

In India, the wild variety of $W$. somnifera is found in three forms.

i. Sandy desert soil of Pilani, Marwar, and some parts of Rajasthan. Root: Straight, thick, unbranched, thick, fleshy, and distinctly fusiform.

ii. Chandigarh and some mountainous areas of Punjab and Uttar Pradesh. Root: A straight unbranched tap root, tapering gradually from the crown, comparable in size and texture to form 1 thick and unbranched.

iii. Near Delhi and Ahmedabad along hedges and shady habitats. Root: branched, light, and woody.

Cultivated in Madhya Pradesh (Manasa). Root straight tap, slender, unbranched or slightly branched, smooth, light coloured and starchy. Grown as an annual crop (Atal, 1975).
In Manasa, the plant height and plant biomass were found to be the highest followed by Hyderabad. Leaves were found to be the principal organ for WS-3 accumulation while roots mainly accumulate WS-1, suggesting a spatial variation of withanolides. Plants of Manasa population alone showed the presence of WS-2. Withanolide accumulation was also the highest in Manasa population, with $1.312 \%$ WS-3 in the leaves and $0.083 \%$ WS- 1 in roots. Withanolide accumulation correlated positively with developmental stages and the highest content of these withanolides was found at maturity in both roots and leaves, indicating that plants be harvested at maturity stage for maximum economic benefit (Mir, 2014).

Till the thirteenth century, the root of the wild variety of Aswagandha was widely used in Ayurveda as a potent sex tonic.

Sharangadhara Samhita attributed the following properties to Ashwagandha:

i. The root should be fresh. Dried root was considered to be ineffective.

ii. While breaking, the root should smell like a horse's urine.

The wild variety was found in places far off Hamirpur of Shakambri State, where it was living. How it was possible to get the drug in a fresh state. We may get some clue if we analyze its synonyms: Ashwaavarohikaa (Ch.Chi 10/35, synonym in Nighantus), Ashwamuutri (Su. U.42/94), Turag gandhaa, Vaajigandhaa, Hayagandhaa, Hyaahwaa.

Ashwa, Turag, Vaaji indicate that it was brought from a far-off location by horse-rider herb collectors. Muutri indicates that the drug was preserved in the horse urine to protect it from drying as it was to be used as a fresh herb. This practice has a valid scientific basis as horse urine contains significant amounts of ammonia. In modern extraction, ammonia is used to enhance the extraction of alkaloids from plants as is done with the extraction of Ashwagandha roots. Thus, it is established that the use of horse urine in transporting the fresh roots has a scientific basis.

Ashwagandha is described in the third Varga of Bhavaprakasha Nighantu, Guduchyaadi Varga. All synonyms of Haya (Horse) were considered as the synonyms of Ashwagandha. Varahkarni, Varda, Balda, Kusthgandhini have been also described in the nighantu. The following karma (an outcome of the drug) have been enumeratedVaata-Kapha pacifier, Balya (anabolic tonic), Rasayana rejuvenating tonic), Atisukrala (Spermatic) and effective in Svitra (leucoderma), kshaya (tuberculosis), Shotha (inflammation). When we screen Bhavaprakasha, we find Ashwagandha with other plant drug in 11 oils, in one avleha (medicinal confection, Kushmaandaavlela), and two churns (powdered drugs) and 11 polyherbal compounds. As a single herb, it was used in 4 prescriptions, as kalka (paste) in two rejuvenating and cardiac tonics, and one qwatha (decoction) for Vandhyaa Chikistaa (for female sterility).

Now, let us review the profile of the horse urine in Ayurveda as its synergistic action with Ashwagandha root also cannot be ignored.

The urine of cow, goat, sheep, she-buffalo, elephant, horse, camel and ass increases pitta and is ruksha (dry), sharp, and hot; it has a katu (pungent) taste along with saltish; it cures krmi (worm infestation), sopha (oedema), udara (diseases of the abdomen 
including ascites), anaaha (constipation), shuula (colic), aruci (anorexia), poison, shvitra (leucoderma), kustha (skin diseases, including leprosy) and arsh (piles). It is dipana (increases the digestive fire), paachana (digests the food), and is bhedi (purgative). Ashtangasangraha, Sutra VI/235-237 (Sarma and Rao, 1980).

The urine of a horse is deepana (digestion-stimulant), katu (pungent), tikshna and ushna (sharp and hot). It cures diseases caused by the aggravation of Vaayu and mind. It alleviates Kapha and kimi (parasitic infection) and dadru (ringworm) (Dash and Singh, 1987).

Horse urine is bitter, pungent, and destroyer of Kustha, wounds and poisons. Horses urine is pungent sharp, hot, pacifies Kapha, stimulates digestive power, alleviates disorders of Vata and mind is useful in worm and ringworm (Kumar and Kashyap, 2017).

Now, let us review the use of Ashwagandha in Sharangadhara Samhita (Thirteenth century).

Ghrit Kaamdeva Ma.Kh. 9/94: Sex tonic.

Rasa Madana Kamadeva Ma. Kh. 12/263: Sex tonic.

Lingavridhow Yonisankoche U. Kh, 11/113: For increasing the size of the penis, also firming the vagina. The paste of fresh root was to be applied topically.

In Bhavaprakasha (Sixteenth century), the prominence of aphrodisiac drugs declined and a new trend emerged to include Ashwagandha in oil. Ashwagandha with other plant drugs entered into 11 oils, and only in one avleha (medicinal confection, Kushmaandaavlela), and two churns (powdered drugs) and 11 polyherbal compounds. As a single herb, it was used in 4 prescriptions, as kalka (paste) in two rejuvenating and cardiac tonics, and one qwatha (decoction) for vandhyaa chikitsaa (female sterility).

The profile of the drug changed. Either the wild species was not available or cultivated species captured the market. Ashwagandha's efficacy as an aphrodisiac drug was also challenged as is evident from a study published in the Asian Journal of Andrology. Moreover, Department of Anatomy, Faculty of Medicine, University of Ruhuna, Sri Lanka, examined the effect of Ashwagandha root extract on the sexual behaviour of male rats. The findings of this experimental study indicated that the use of Ashwagandha roots may be detrimental to male sexual competence. Male rats were orally administered $300 \mathrm{mg} / \mathrm{kg} /$ day of root methanolic extract for 7 days. Their sexual behaviour was evaluated 7 days before the treatment, on day 3 and day 7 of treatment and day 7, 14, and 30 post-treatment by pairing each male with a receptive female. The root extract induced a marked impairment in libido, sexual performance and sexual vigour. It also caused penile erectile dysfunction. These effects were partly reversible on cessation of treatment. These antimasculine effects were not due to changes in testosterone levels or toxicity, but might be attributed to hyperprolactinemic, GABAergic, serotonergic or sedative activities of the extract (Ilayperuma et al., 2002).

In 'Ayurvedic Pharmacopoeia of India' (Part I, Vol. I), Ashwagandha (so-called Withania somnifera) has been recommended (based on classical references) in the following conditions:

Klaibya (male impotence)
Kshaya (phthisis)

Daurbalya (weakness)

Vaataroga (diseases of the nervous system/neurological diseases)

Shoth (inflammation, oedema).

(English equivalents are quoted from 'The Ayurvedic Formulary of India, Part I, Second Revised English Edn., 2003, 461-480.)

Out of these, only the last two survived. The first two are being touted for business as the whole world is crazy to try anything and everything which is an aphrodisiac. Ashwagandha root used in India smells woody with characteristic odor with a bitter note.

\section{Review the morphology and constituents of cultivated Ashwagandha}

Ashwagandha (W. somnifera): An improved strain WSR is developed and released for commercial cultivation by IIIM, Jammu. The dry biomass yield of this newly developed variety shows a $200 \%$ increase in yield over the conventionally grown varieties

Chetak (Naguri Withania, CIMAP variety): A semi vigorous, medium green small leaves size and whitish-green stem, was found to be highly promising for high dry root yield $(11.77 \mathrm{ql} / \mathrm{ha} \mathrm{v} / \mathrm{s}$ check $5.45 \mathrm{ql} / \mathrm{ha})$ with high total withanolide content $(0.40 \mathrm{v} / \mathrm{s} 0.20 \%$ in check). The fresh and dry leaf yield was also high (1.722 and $0.453 \mathrm{ql} / \mathrm{ha} \mathrm{v} / \mathrm{s} 0.872$ and $0.147 \mathrm{ql} / \mathrm{ha}$ in check) with high withaferine content $1.223 \mathrm{v} / \mathrm{s} 0.788 \%$ in the check.

Pratap (CIMAP variety): A highly vigorous, dark green medium size leaves and dark green stem, highly promising for high dry root yield (34.95 ql/ha v/s Poshita $21.99 \mathrm{ql} / \mathrm{ha}$ ) with high total withanolide content $(0.31 \mathrm{v} / \mathrm{s} 0.25 \%$ in Poshita). The fresh and dry leaf yield was also high (5.39 and $0.87 \mathrm{ql} / \mathrm{ha} \mathrm{v} / \mathrm{s} 2.83$ and $0.50 \mathrm{ql} / \mathrm{ha}$ in Poshita) with high withaferin content in dry leaves was $0.720 \mathrm{v} / \mathrm{s} 0.528 \%$ in the check variety Poshita.

Poshita (South India, specially Anantnag): Amedium tall, semi broad, medium-dark colour leaf with red coloured berries. The estimated dry root yield was $14 \mathrm{q} / \mathrm{hav} / \mathrm{s}$ check which is $8 \mathrm{q} / \mathrm{ha}$. Total withanolides $(\mathrm{kg} / \mathrm{h})$ is 0.25 . It is a popular variety in South India.

Nmitli 118: New chemotypes identified under a NMITLI(New India Millennium Indian Technology Leadership Initiative) project effort was made to collect the Ashwagandha germplasm, which resulted into the collection of 150 independent accessions from various geographical locations, with many of them having contrasting chemotypes. Efforts are underway to explore the pharmacological activities of selected chemotypes and individual molecule to identify the best chemotype for adaptogenic activity. Other varieties are as Jawahar, WS 20 and tall Ashwagandha (Srivastava et al., 2018).

Poshita and Rakshita are high yielding varieties released by CSIRCIMAP, Lucknow. Jawahar-20 is cultivated in Madhya Pradesh. WSR is another variety released by CSIR-Regional Research Laboratory, Jammu. Nagori is a local variety with starchy roots.

\subsection{Root grades}

A grade

Root pieces up to $7 \mathrm{~cm}$ in length, $1-1.5 \mathrm{~cm}$ in diameter, solid cylindrical with smooth external surface and pure white from inside. 


\section{B grade}

Root pieces up to $5 \mathrm{~cm}$ in length, $1 \mathrm{~cm}$ or less in diameter, solid, brittle and white from inside.

\section{C grade}

Solid root pieces up to 3-4 $\mathrm{cm}$ in length, $1 \mathrm{~cm}$ or less in diameter.

D grade

Small root pieces, semisolid or hollow, very thin, yellowish inside and $<1 \mathrm{~cm}$ in diameter of late farmers are grading the roots into 7 or more grades.

\section{A comparative study of wild and cultivated Ashwagandha}

A study was conducted at Birsa Agricultural University, Kanke, Ranchi, in 2011, to evaluate the comparative alkaloid content of wild and cultivated germplasm of Ashwagandha ( $W$. somnifera). The wild germplasm was collected from Khunti forest area and the cultivated germplasm are JA-20, JA-134, Poshita.

The experiment was conducted in Randomized Block Design (RBD) to know the growth and yield parameters of different germplasm of the Ashwagandha. The experiment was replicated five times. The total numbers of treatments were four. The total area of the plot was $4 \mathrm{~m} \mathrm{X} 3 \mathrm{~m}$ and the number of the plant in each plot was 100 . The number of plants to be used for the collection of data from each plot was ten (randomly).

The growth parameters of Ashwagandha like plant height $(\mathrm{cm})$, number of main branches, number of berries per plant, leaf length $(\mathrm{cm})$, days to flower initiation, days to seed harvest, and the yield parameters like number of seeds per berry, seed yield per plant (gm) were recorded. The yield parameters of Ashwagandha like main root length $(\mathrm{cm})$, main root diameter $(\mathrm{cm})$, number of secondary roots per plant, fresh root weight per plant (gm), dry root weight per plant (gm) were taken on final harvesting. The alkaloid content (\%) and fiber content (\%) in root are estimated through HPLC method in the laboratory. Average value of $\mathrm{pH}$, organic carbon, available nitrogen, phosphorus, and potassium of the soil of research area was estimated before plantation of Ashwagandha. Maximum plant height among the germplasm was found in Poshita, i.e., $98.00 \mathrm{~cm}$ whereas minimum height obtained in wild variety, i.e., $68.40 \mathrm{~cm}$. Maximum number of berries, 137.40 , per plant among the germplasm was found in Poshita, whereas minimum number of berries per plant, 104.00, was obtained in wild variety. Maximum days to flower initiation and days to seed harvest among the germplasm was found in Poshita. Maximum number of seeds, 37.80 , per berry among the germplasm was found in JA-134, whereas the minimum number of seeds, 34.80 , per berry was obtained in wild germplasm. Maximum main root diameter $(21.26 \mathrm{~mm})$ among the germplasm was found in Poshita, whereas minimum main root diameter $(13.00 \mathrm{~mm})$ was obtained in wild variety.

The number of secondary root per plant at the harvesting stage varied from 2.04 to 5.32. Maximum fresh root weight per plant (38.84 gm) among the germplasm was found in Poshita, whereas minimum fresh root weight per plant (20.80 gm) obtained in wild germplasm. Dry root weight per plant at the harvesting stage varied from $6.84 \mathrm{gm}$ to $12.88 \mathrm{gm}$. Out of four germplasm of Ashwagandha, maximum dry root weight per plant was 12.88 gm in Poshita, whereas minimum dry root weight per plant obtained in wild germplasm was $6.84 \mathrm{gm}$. The alkaloid content (\%) in root varied from $0.20 \%$ to $0.35 \%$. Maximum alkaloid content in root among the germplasm was found in JA-134, whereas minimum alkaloid content $(\%)$ in root obtained in wild germplasm. The fiber content (\%) in root varied from $18.76 \%$ to $32.04 \%$. Maximum fiber content (\%) in root among the germplasm was found in JA-134, whereas minimum fiber content $(\%)$ in root obtained in wild germplasm. It is concluded that for the production of root and seed, Poshita variety may be adopted for commercial cultivation under the climatic conditions of Jharkhand. From the active principles point of view, JA-134 variety may be adopted, because its recorded value was found significantly superior than other variety (Lal, 2013).

\section{DNA barcoding of $W$. somnifera and $W$. ashwagandha}

Based on morphology, chemical profiling, crossing ability, amplified fragment length polymorphism and comparison of nucleotide sequences of nuclear ribosomal internal transcribed spacer (ITS), the cultivated form of $W$. somnifera, a species of therapeutic value, has been circumscribed as a new species, $W$. ashwagandha. The present study was undertaken to ascertain whether the two species can be distinguished on the basis of DNA barcoding. Six barcode loci, ITS, ITS2, matK (maturase K), rbcL (ribulose-bisphosphate carboxylase/oxygenase, large subunit), rpoC1 (RNA polymerase- $\beta 2$ subunit, main catalytic subunit) and trnH-psbA spacer (transfer RNA for histidine-photosystem II protein D1 spacer) from $W$. somnifera, W. ashwagandha, their hybrid, and 'ashwagandha' market samples were amplified, sequenced, and compared. ITS, ITS2 and matK distinguished two species on the basis of phylogenetic tree method. Likewise, BLAST 1 analysis based on ITS, ITS2, matK, and rbcL individually discriminated two species. However, on the basis of Kimura 2 parameter distances, two species could not be distinguished as the requirement of a distinct barcode gap-the highest intraspecific distance being lower than the lowest interspecific distance was not met by any of the loci. If compared by character-based method, ITS, ITS2 and matK sequences of the two species had distinct diagnostic nucleotides (pure character attributes) at nine, four and one positions, respectively. Interestingly, all market samples co-segregated and shared character attributes with $W$. somnifera (Malik et al., 2017).

\section{Pharmacological activity}

\subsection{Neuroprotective activity}

A study was carried out in CSIR-Indian Institute of Integrative Medicine, Sanat Nagar, Srinagar, India, to evaluate withanoneinduced excitotoxicity in retinoic acid, differentiated Neuro2a cells. Cells were pre-treated with 5,10 and $20 \mu \mathrm{M}$ doses of withanone and then exposed to 3-mM NMDA for $1 \mathrm{~h}$. MK801, a specific NMDA receptor antagonist, was used as positive control. The results indicated that NMDA induces significant death of cells by the accumulation of intracellular $\mathrm{Ca}^{2+}$, generation of reactive oxygen species (ROS), loss of mitochondrial membrane potential, crashing of $\mathrm{Bax} / \mathrm{Bcl}-2$ ratio, release of cytochrome $\mathrm{c}$, increased caspase expression, induction of lipid peroxidation as measured by malondialdehyde levels and cleavage of poly (ADP-ribose) 
polymerase-1 (Parp-1), which is indicative of DNA damage. All these parameters were attenuated with various doses of withanone pre-treatment. These results suggest that withanone may serve as potential neuroprotective agent (Dar et al., 2017).

\subsection{Withanolide-A and immune responses}

The effects of graded doses of a chemically standardized aqueous alcoholic (1:1) root extract (AGB) of $W$. somnifera on the immune system of SRBC immunized BALB/c mice were investigated. Mice were administrated AGB orally for 15 days. AGB stimulated cell mediated immunity, IgM and IgG titers reaching peak value with $30 \mathrm{mg} / \mathrm{kg}$ b.wt. Flow cytometric analysis of lymphocyte surface markers of $\mathrm{T}$ cells $(\mathrm{CD} 3(+), \mathrm{CD} 4(+)$ and $\mathrm{CD} 8(+))$ and $\mathrm{B}$ cells $(\mathrm{CD} 19(+))$ indicated prominent enhancement in proliferation and differentiation of lymphocytes. The extract selectively, induced type 1 immunity because it guided enhanced expression of $\mathrm{T}$ helper cells (Th) 1 cytokines interferon (IFN)-gamma and interleukin (IL)-2 while Th 2 cytokine IL-4 observed a moderate decline. Confirmation of Th1 polarization was obtained from augmented levels of IgG2a over IgG1 in the blood sera of AGB treated groups. Withanolide-A, a major constituent of AGB appeared responsible for Th1 skewing effect of the extract as it significantly increased the levels of Th1 cytokines, decreased moderately IL-4 and significantly restored the selective dexamethasone inhibition of Th1 cytokines in mouse splenocytes cultures in vitro. In addition, AGB also strongly activated macrophage functions ex vivo and in vitro indicated by enhanced secretion of nitrite, IL-12 and TNF-alpha. In contrast, IL-10 remained unchanged again suggesting that AGB critically influenced Th1 profile of the cytokines. The studies suggested that AGB supports predominantly Th1 immunity with increase in macrophage functions. The standardized root extract of no toxicological consequences might, therefore, find useful applications against the intracellular pathogens and in the management of immunesuppressed diseases (Malik et al., 2007).

\subsection{Antitumor and radiosensitizing effects}

Antitumor and radio sensitizing effects of alcoholic root extract of W. somnifera and their modification by heat were studied in vivo on Sarcoma-180 grown on the dorsum of adult BALB/c mouse. Ashwagandha (AT) was injected (ip) at a dose of $500 \mathrm{mg} / \mathrm{kg}$ body wt for 10 consecutive days into mouse bearing tumor of $50 \pm 5 \mathrm{~mm}$ [sup 3], with or without a local treatment of 10 Gy gamma radiation (RT) or hyperthermia at $43^{\circ} \mathrm{C}$ for $30 \mathrm{~min}$ (HT) or both on 5th day of AT. The response was assessed on the basis of tumor regression, growth delay, animal survival and changes in the tumor glutathione content. Ashwagandha, RT and HT individually produced 18,38 and $45 \%$ complete response (CR), respectively, but RT gave the best long term survival. Ashwagandha increased the effect of radiation on tumor regression as well as growth delay, but $\mathrm{AT}+\mathrm{HT}$ gave a better tumor cure. However, both these combinations gave almost identical long term survival, which was not much higher than that produced by RT alone. The combination of Ashwagandha for 10 days with one local exposure to RT followed by HT significantly increased the tumor cure, growth delay of partially responding tumors and animal survival. The combination also significantly and synergistically depleted the tumor GSH level, with no recovery even at $3 \mathrm{~h}$ after treatment. It is concluded that Ashwagandha, in addition to having a tumor inhibitory effect, also acts as a radiosensitizer and heat enhances these effects. The severe depletion in the tumor glutathione content by the combination treatment must have enhanced the tumor response, as the inherent protection by the thiol will be highly reduced (Devi et al., 1993).

\subsection{Antistress activity}

The antioxidant activity of $W$. somnifera glycowithanolides was assessed in chronic footshock-induced stress. The stress procedure, given once daily for 21 days, induced an increase in activity of superoxide dismutase (SOD) and lipid peroxidation (LPO), with concomitant decrease in activities of catalase and glutathione peroxidase in both the brain regions. Administration of $W$. somnifera extract in the doses of 10,20 and $50 \mathrm{mg} / \mathrm{kg}$ orally $1 \mathrm{~h}$ prior to the stress procedure for 21 days produced a dose-related reversal of the stress effects. In another study, the effect of $W$. somnifera root extract was seen in stress-induced neuronal degeneration in rats. The ultrastructural study of neuronal cell bodies in hippocampal sublayer (CA1-CA4) was studied. Study suggested the cytoprotective effect of $W$. somnifera in improving degenerating characteristics (which included karyorrhexis, membrane blebbing, chromatin condensation, chromatin fragmentation and intracellular spacing) in rat brain. Two glycosides, sitoindoside VII and sitoindoside VIII isolated from the roots of $W$. somnifer $a$ showed significant antistress activity when tested in diverse spectrum of stress-induced paradigms. Similarly, sitoindoside IX and sitoindoside X in a dose range of 50-200 mg/kg p.o. produced significant antistress activity in mice and rats and augmented learning acquisition and memory retention in both young and old rats (Kulkarni and Dhir, 2008).

\subsection{Adaptogenic activity of withanolide-free aqueous fraction}

A new withanolide-free hydrosoluble fraction was isolated from the roots of $W$. somnifera and was evaluated for putative antistress activity against a battery of tests to delineate the activity of this fraction. The latter fraction exhibited significant antistress activity in a dose-related manner and was further studied against chemical and physical induced stress in rats and mice. The extract of $W$. somnifera root (a commercial preparation) was also used to compare the results. A preliminary acute toxicity study in mice showed a good margin of safety with a high therapeutic index (Singh et al., 2003).

\subsection{Anticancer activity of $W$. somnifera}

Withanolides are a large group of steroidal lactones found in Solanaceae plants that exhibit potential anticancer activities (Wang et al., 2012), have demonstrated that a withanolide, tubocapsenolide A, induced cycle arrest and apoptosis in human breast cancer cells, which was associated with the inhibition of heat shock protein 90 (Hsp90). The researchers further investigated other withanolides capable of inhibiting Hsp90 and analyzed the structure-activity relationships, nine withanolides with different structural properties were tested in human breast cancer cells MDA-MB-231 and MCF-7 in the present study. The data demonstrate that the 2,3-unsaturated double bond-containing withanolides inhibited $\mathrm{Hsp} 90$ function, as evidenced by selective depletion of Hsp90 client proteins and induction of Hsp70. The inhibitory effect of the withanolides on Hsp90 chaperone activity was further confirmed using in vivo heat shock luciferase activity recovery assays. Importantly, Hsp90 
inhibition by the withanolides was correlated with their ability to induce cancer cell death. In addition, the withanolides reduced constitutive NF- $\kappa \mathrm{B}$ activation by depleting I $\kappa \mathrm{B}$ kinase complex (IKK) through inhibition of Hsp90. In estrogen receptor (ER)-positive MCF-7 cells, the withanolides also reduced the expression of ER, and this may be partly due to Hsp90 inhibition. Taken together, the results suggest that Hsp90 inhibition is a general feature of cytotoxic withanolides and plays an important role in their anticancer activity.

Gu et al. (2017) showed that withaferin A(WA), a naturally occurring steroidal lactone, directly binds to Hsp90 and leads to the degradation of Hsp90 client protein. The researchers investigated the structure activity relationship (SAR) of withanolides for their inhibition of Hsp90 and anti-proliferative activities in pancreatic cancer cells. In pancreatic cancer Panc-1 cells, withaferin A (WA) and its four analogues withanolide E (WE), 4-hydroxywithanolide E (HWE), 3-aziridinylwithaferin A (AzWA) inhibited cell proliferation with IC50 ranged from 1.0 to $2.8 \mu \mathrm{M}$. WA, WE, HWE, and AzWA also induced caspase-3 activity by 21-, 6-, 11- and 15fold, respectively, in Panc-1 cells, while withaperuvin (WP) did not show any activity. The data showed that WA, WE, HWE, and AzWA, but not WP, all directly bound to Hsp90 and induced Hsp90 aggregation, hence inhibited Hsp90 chaperone activity to induce degradation of Hsp90 client proteins Akt and Cdk4 through proteasome-dependent pathway in pancreatic cancer cells. However, only WA, HWE and AzWA disrupted Hsp90-Cdc37 complexes but not WE and WP. SAR study suggested that the C5(6)-epoxy functional group contributes considerably for withanolide to bind to Hsp90, inhibit Hsp90 chaperone activity, and result in Hsp90 client protein depletion. Meanwhile, the hydroxyl group at C-4 of ring A may enhance withanolide to inhibit Hsp90 activity and disrupt Hsp90-Cdc37 interaction. These SAR data provide possible mechanisms of anti-proliferative action of withanolides (Yadav et al., 2017).

\section{Toxicological activity}

\subsection{Cytotoxic potential of modified ring A of withaferin A}

Regio-/stereoselective Michael addition to ring A of withaferin-A was performed using an optimized reaction procedure to synthesize a library of 2,3-dihydro,3- $\beta$-substituted withaferin-A derivatives. The analogues, thus obtained were evaluated for in vitro cytotoxicity against various human cancer cell lines. 3-Azido analogue exhibited 35-fold increase $(\operatorname{IC}(50)=0.02-1.9 \mu \mathrm{M})$ in cytotoxicity against almost the entire cell lines tested when compared to the parent molecule. However, further modifications of 3-azido analogue with various alkynes under Husigen's cycloaddition conditions generated a variety of triazole derivatives with reduced cytotoxicity (Yousuf et al., 2011).

QSAR and molecular docking based prediction of withanolide analogs showed that 2,3-dihydrowithaferin A-3-beta- $O$-sulfate, withanolide 5, withanolide A, withaferin A, CID_10413139, CID_11294368, CID_53477765, CID_135887, CID_301751 and CID_3372729 against Sk-Br-3 and CID_73621, CID_435144, CID_301751 and CID_3372729 possess a significant anticancer activity against the MCF7/BUS. The QSAR results for SK-Br-3 suggested that connectivity index (order 0 , standard), dipole vector X (Debye), molar refractivity, shape index (basic kappa, order 2), whereas for MCF7/BUS, atom count (all atoms), dielectric energy ( $\mathrm{kcal} / \mathrm{mole}$ ), total energy (hartree) and heat of formation ( $\mathrm{kcal} / \mathrm{mole}$ ) correlated well with the activity. In docking studies, active withanolide analogs showed high binding affinity against $\beta$-tubulin receptor protein. The withanolide analogs CID_301751 and CID_3372729 showed good predicted activity and binding affinity to $\beta$-tubulin receptor protein. The docking results showed that the major influencing factors of molecular interactions between withanolide analogs and $\beta$-tubulin were $\mathrm{H}$-bonds and hydrophobic and electrostatic interactions. The in silico prediction of oral bioavailability (rule of five) and ADMET risk profiling were within their acceptable limit for active analogs. These compounds have rationalized the structural requirement and need further lead optimization for designing of novel $\beta$-tubulin inhibitors (Yadav et al., 2017; Gu et al., 2014).

\section{Cultivated varieties of $W$. somnifera, a possible drug for Alzheimer}

Withanoloids isolated from the $W$. somnifera are known to inhibit acetylcholinesterase and butylcholinesterase in a dose dependent manner. The cholinesterase inhibitory potential along with calcium antagonistic ability has made $W$. somnifera a possible drug candidate to treat Alzheimer's disease and associated problems. Sitoindosides VII-X, and withaferin-A, isolated from aqueous methanol extract from the roots of cultivated varieties of $W$. somnifera were used to attenuate cerebral functional deficits, including amnesia, in geriatric patients. The effect of these active constituents of $W$. somnifera was also investigated for putative nootropic activity in a experimentally validated Alzheimer's disease model. The syndrome was induced by ibotenic acid lesioning of the nucleus magnocellularis in rats. W. somnifera significantly reversed both ibotenic acid induced cognitive deficit and the reduction in cholinergic markers after 2 weeks of treatment. Orally administrated withanoside IV may ameliorate neuronal dysfunction in Alzheimer's disease due to sominone, an aglycone of withanoside IV. Similarly, withanolide-A ( 1 microM) induces significant regeneration of both axons and dendrites, in addition to the reconstruction of pre-and postsynapses in the neurons. and therefore, may prove an important candidate for the therapeutic treatment of neurodegenerative diseases (Choudhary et al., 2005).

\section{Root extracts and its reported activity}

8.1 Alcoholic extract: Neurological, radiosensitizer, anticonvulsant, anti-inflammatory, sedative, antitumor, antibacterial.

8.2 Methanolic extract: GABA mimetic activity GABA receptor mediates anticonvulsant activity, protective effect as amygdaloid kidlling anti-inflammatory ( $70 \%$ extract), antistress.

8.3 Chloroform-methanol extract: Prevention of Alzheimer's disease (Sehgal et al., 2012), immunom-odulatory, antiinflammatory, nematicidal, hepatoprotective.

8.4 Water extract: Nephroprotective, antistress, antianxiety, hypothyroidism, anticonvulsant, anti-inflammatory, antiarthritic, hepatoprotective.

8.5 Petroleum ether extract: Insecticidal (Mir et al., 2012). 


\section{Conclusion}

W. somnifera $\mathrm{sp}$. Novo should not be considered a substitute for classical Ashwagandha. The description of aphrodisiac uses in Ayurvedic classical texts and the name Ashwagandha proved a great market puller world over. Now, as the profile of Ashwagandha has changed, the drug should be marketed based on the evidence of its active principles and specific biologicals should be disclosed. It should be treated as a new drug.

\section{Conflict of interest}

The authors declare that there are no conflicts of interest relevant to this article.

Table 2: Major phytochemical constituents of $W$. somnifera and related pharmacological activity

\begin{tabular}{|c|c|c|c|}
\hline Active metabolites & Group of metabolites & Pharmacological activities & References \\
\hline Anaferine & \multirow[t]{9}{*}{ Alkaloid } & Excitotoxicity and neurodegenerative disorders. & \multirow{14}{*}{$\begin{array}{l}\text { Tursunova, et al., 1993; } \\
\text { Tursunova, et al., } 1977\end{array}$} \\
\hline Anahygrine & & nAChR agonist & \\
\hline Cuscohygrine & & CNS activity & \\
\hline Pseudotropine & & Anticholinergic. & \\
\hline \begin{tabular}{|l} 
Somniferinine \\
\end{tabular} & & \begin{tabular}{|l|} 
Hypotensive \\
\end{tabular} & \\
\hline Somniferiene & & Hypnotic & \\
\hline Tropanol & & anticholinergic & \\
\hline Withanin & & Sedative & \\
\hline Withananine & & \begin{tabular}{|l|} 
Sedative \\
\end{tabular} & \\
\hline Chlorogenic acid & Polyphenol & Hypotensive, antioxidant & \\
\hline Cysteine & Amino Acid & Antioxidant & \\
\hline Scopoletin & Coumarin & Anti-inflammatory & \\
\hline Withanolides A-Y & Steroidal lactones & $\begin{array}{l}\text { Immunomodulatory, anti-inflammatory, antiarthritic, } \\
\text { angiogenesis inhibitor, anticholinesterase, antioxidant, }\end{array}$ & \\
\hline Withanamides A-I & Indolyl metabolite & Antialzheimer's & \\
\hline Ashwagandhanolide & Steroidal lactone & Anticancer & \multirow[t]{2}{*}{ Jayaprakasam et al., 2004} \\
\hline$\beta$-Sitosterol & Plant sterol & Anti hyperlipidemic & \\
\hline Withaferin $\mathrm{A}$ & Steroidal lactone & Anticancer & \multirow{7}{*}{$\begin{array}{l}\text { Kapoor, 2011; } \\
\text { Gupta and Rana, } 2007\end{array}$} \\
\hline Choline & Acetylcholine precursor & Anxiolytic, hepatoprotective. & \\
\hline Pseudowithanin & Alkaloid & Hypotensive, bradycardiac & \\
\hline \begin{tabular}{|l|} 
Pseudotropine \\
\end{tabular} & Alkaloid & Anticholinergic & \\
\hline dl-isopelletierine & Alkaloid & Anthelminthic & \\
\hline Nicotine & Alkaloid & Stimulant. & \\
\hline $3 \alpha$-tigloyloxytropane & Alkaloid & Anti-microbial, antioxidant, CNS & \\
\hline Sitoindosides VII-X & Steroidal Saponins & Antistress & Ghosal et al., 1989 \\
\hline
\end{tabular}

\section{References}

Abdeljebbar L.H. (2009). Effects of withanolides from Withania adpressa, Therapie, 64(2):121-127.

Alfonso, D.; Bernardinelli, G and Kapetanidis, I. (1993). Withanolides from Lochroma coccineum. Phytochemistry, 34:517-521.

Anonymous. http://agritech.tnau.ac.in/horticulture/horti_medicinal\% 20crops_aswagandha.html

Atal C.K. (1975). Pharmacognosy and phytochemistry of Withania somnifera, CCRIMH, New Delhi. Now CCRAS.

Choudhary, M.I.; Nawaz, S.A.; Haq, Z.; Lodhi, M.A.; Ghayur, M.N.; Jalil, S.; Riaz N.; Yousuf, S.; Malik, A.; Gilani, A.H and Rahman, A. (2005). Withanolides, a new class of natural cholinesterase inhibitors with calcium antagonistic properties. Biochem. Biophys. Res. Commun., 334(1):276-287.

Dar, N.J.; Bhat, J.A.; Satti, N.K.; Sharma, P.R.; Hamid, A. and Ahmad, M. (2017) Withanone, an active constituent from W. somnifera, affords protection against NMDA-induced excitotoxicity in neuron-like cells. Mol. Neurobiol., 54(7):5061-5073.

Dash, V.B. and Kashyap, V.L. (1997). Materia Medica of Ayurveda based on Ayurveda Saukhyam of Todaraananda (Sixteenth century). pp:206.
Dermarderosian, A. (2004). The review of natural products: Formerly lawrence review of natural products published by facts and comparisons. Edition 4.

Devi, P.U.; A.C. Sharada, A.C. and Solomon and F.E. (1993). Antitumor and radiosensitizing effects of Withania somnifera (Ashwagandha) on a transplantable mouse tumor, Sarcoma-180. Indian Journal of Experimental Biology, 31(7):607-611.

Ghosal, S.; Lal, J.; Srivastava, R.; Bhattacharya, S.K.; Upadhyay, S.N.; Jaiswal, A.K. and Chattopadhyay, U. (1989). Immunomodulatory and CNS effects of sitoindosides IX and X, two new glycowithanolides from Withania somnifera. Phytotherapy Research, 3(5):201-206.

Gu, M.; Yu, Y.; Gunaherath, G.M.; Gunatilaka, A.A.; Li, D. and Sun, D. (2014). Structure-activity relationship (SAR) of withanolides to inhibit Hsp90 for its activity in pancreatic cancer cells. Invest New Drugs, 32(1):68-74.

Gupta, G.L. and Rana, A.C. (2007). Withania somnifera (Ashwagandha): A review. Pharmacog. Rev., 1:129-136.

Husain, A. (1992). Dictionary of Medicinal Plants, CIMAP, pp:494.

Ilayperuma, I.; Ratnasoorya, W.D. and Weerasoorya. T.R. (2002). Effect of Withania somnifera root extract on the sexual behaviour of male rats. Asian J. Androl., 4(4):295-298. 
Jayaprakasam, B.; Strasburg, G.A. and Nair, M.G. (2004). Potent lipid peroxidation inhibitors from Withania somnifera fruits. Tetrahedron, 60:3109-3121.

Kapoor, L.D. (2001). Handbook of Ayurvedic medicinal plants; CRC Press: London, UK, pp:337-338.

Kulkarni, S.K. and Dhir, A. (2008). Withania somnifera: An Indian ginseng. Prog. Neuropsychopharmacol. Biol. Psychiatry, 32(5):1093-1105.

Kumar, B. and Singh, J. (2017). Concept of urine in brihattrayi. International Journal of Ayurveda and Pharma Research, 6(11): 14.

Lal, M. (2013). Evaluation of wild and cultivated germplasm of Withania somnifera (L.) Dunal (Ashwagandha) (Thesis). Birsa Agricultural University, Kanke, Ranchi, Jharkhand.

Malik, F.; Singh, J.; Khajuria, A.; Suri, K.A.; Satti, N.K.; Singh, S.; Kaul, M.K.; Kumar, A.; Bhatia, A. and Qazi, G.N. (2007). A standardized root extract of Withania somnifera and its major constituent withanolide-A elicit humoral and cell-mediated immune responses by up regulation of Th1-dominant polarization in BALB/c mice. Life Sciences, 80:1525-1538.

Malik, S.; Mir, B.A.; Singh, H.K.; Chadhary, M. and Raina, S.N. (2017) DNA barcodes distinguish Withania somnifera and Withania ashwagandha. Proc. Natl. Acad. Sci., India, Sect. B. Biol. Sci., 88: $1413-1424$.

Mir, B.A.; Jabeena, J.K.; Rehman, K.K.; Rehman, H.K. and Koul, S. (2014) Withanolides array of Withania ashwagandha sp. novo populations from India; Sushma Koul. Industrial Crops and Products, 59:9-13.
Mir, B.A.; Khazir, J.; Mir, N.A.; Hasan, T and Sushma Koul, S. (2012). Indian Journal of Drugs and Diseases, 1(6):2278-2958.

Sharma, C.R.R. and Rao, B.M. (1980). Urine in ancient Ayurvedic literature. Bull Indian Inst. Hist. Med., 10(1-4):48-52.

Singh, B.; Chandan, B.K. and Gupta, D.K. (2003). Adaptogenic activity of a novel withanolide-free aqueous fraction from the roots of Withania somnifera Dun. (Part II). Phytother. Res., 17(5):531-536.

Srivastava, M.P.; Gupta, S.; Dixit, S. and Sharma, Y.K. (2018). Withania somnifera (Ashwagandha): A wonder herb with multiple medicinal properties. Asian Journal of Pharmacy and Pharmacology, 4(2): $123-130$

Tursunova, R.N.; Maslennikova, V.A. and Abubakirov, N.K. (1977). Withanolides in the vegetable kingdom. Chem. Nat. Comp., 13:131-138.

Wang, H.C.; Tsai, Y.L and Wu, Y.C. (2012). Withanolides-induced breast cancer cell death is correlated with their ability to inhibit heat protein. PLoS One, 7(5):e37764.

Yadav, D.K.; Kumar. S.; Saloni, Singh, H.; Kim, M.H.; Sharma, P.; Misra, S and Khan F. (2017). Molecular docking, QSAR and ADMET studies of withanolide analogs against breast cancer. Drug Des. Devel. Ther., 11:1859-1870

Yousuf, S.K., Majeed, R., Ahmad, M., Sangwan, P.I., Purnima, B., Saxsena, A.K., Suri, K.A., Mukherjee, D. and Taneja, S.C. (2011). Ring A structural modified derivatives of withaferin $\mathrm{A}$ and the evaluation of their cytotoxic potential. Steroids, 76(10):1213-1222.

Citation C.P. Khare and Andrew Vikram Naharwar (2020). Ashwagandha (Withania somnifera (L.) Dunal): A scientific review with respect to Ayurvedic perspectives. Ann. Phytomed., 9(2):134-141. http://dx.doi.org/10.21276/ap.2020.9.2.11 